\title{
Genotype-phenotype Analysis of Paraoxonase 1 in Schizophrenic Patients Treated with Atypical Antipsychotics
}

\author{
Denis Pavăl ${ }^{1}$, Bogdan Nemeş ${ }^{2}$, Răzvan L. Rusu', Eleonora Dronca ${ }^{1}$ \\ ${ }^{1}$ Department of Molecular Sciences, Faculty of Medicine, Iuliu Hațieganu University of Medicine and Pharmacy, ${ }^{2}$ Third Psychiatry Clinic, \\ Emergency County Hospital, Cluj-Napoca, Romania
}

\begin{abstract}
Objective: Recent studies suggest a possible involvement of low paraoxonase 1 (PON1) enzyme activities in the association between schizophrenia, treatment with atypical antipsychotics and increased cardiovascular (CVD) risk. In the present study, we aimed at investigating the PON1 status in a group of schizophrenic patients treated with either olanzapine or other antipsychotic, as compared to a group of healthy control participants.

Methods: We assessed the arylesterase (AREase) and paraoxonase (POase) activities of PON1, as well as three common polymorphisms of PON1 gene (Q192R, L55M, - 108C >T).

Results: We found significantly lower $(-13.3 \%)$ AREase activity in schizophrenic patients, along with significantly lower $(-18.2 \%)$ POase activity in olanzapine-treated patients with QQ genotype. Furthermore, we found a significant difference between groups in L55M polymorphism distribution, whereas Q192R and $-108 \mathrm{C}>\mathrm{T}$ polymorphisms distributions were similar.

Conclusion: We identified the olanzapine-treated patients with QQ genotype as having the lowest PON1 (POase) activity, providing a possible way of identifying schizophrenic patients exposed to the greatest risk of CVD.
\end{abstract}

KEY WORDS: Paraoxonase 1; PON1, Polymorphism; Atypical antipsychotics; Olanzapine; Cardiovascular diseases.

\section{INTRODUCTION}

Schizophrenia is a severe and chronic mental disorder which often requires lifelong treatment with antipsychotics. ${ }^{1}$ Atypical antipsychotics (AAP) are preferred to conventional antipsychotics in the treatment of schizophrenia, due to their lower risk of extrapyramidal effects and superior therapeutic effect. ${ }^{2)}$ Despite the fact that AAP have significantly improved the quality of life for schizophrenic patients, these drugs are associated with adverse metabolic effects, which may lead to serious cardiovascular disease (CVD). ${ }^{3,4)}$ Among the AAP, olanzapine has the highest potential of inducing adverse metabolic effects. ${ }^{5)}$ Indeed, schizophrenic patients treated with AAP

Received: October 4, 2016/Revised: November 16, 2016 Accepted: December 16, 2016

Address for correspondence: Eleonora Dronca, MD, PhD Department of Molecular Sciences, Faculty of Medicine, luliu Hațieganu University of Medicine and Pharmacy, 6 Louis Pasteur, Cluj-Napoca 400349, Romania

Tel: +40-740198218, Fax: +40-264597257

E-mail: eleonora.dronca@umfcluj.ro are a high-risk group for developing CVD, but the mechanisms underlying this association remain elusive. ${ }^{4,6)}$ However, one key element linking schizophrenia, AAP treatment and increased CVD risk could be the paraoxonase 1 (PON1) enzyme.

Human PON1, encoded by the PON1 gene on chromosome 7q21.3, is a high-density lipoprotein-associated enzyme, which exerts a broad range of hydrolytic activities. ${ }^{7)}$ Traditionally assayed are the arylesterase (AREase) and the paraoxonase (POase) activities. ${ }^{8)}$ PON1 concentration and activities display extreme interindividual variation, due to PON1 gene polymorphisms. ${ }^{9)}$ The $P O N 1$ gene contains three common polymorphisms: Gln192Arg (Q192R, rs662) and Leu55Met (L55M, rs854560) in the coding region, and $-108 C>T($ rs705379) in the promoter. ${ }^{10)}$ The Q192R polymorphism has a great influence on the POase activity, rendering a low activity in subjects with QQ genotype and high activity in subjects with QR and RR genotypes. ${ }^{9,11)}$ However, it does not influence the AREase activity, which is considered a reliable indicator of PON1 concentration. ${ }^{12)}$ The L55M poly-

(ㄷ) This is an Open-Access article distributed under the terms of the Creative Commons Attribution Non-Commercial License (http://creativecommons.org/licenses/by-nc/4.0) which permits unrestricted non-commercial use, distribution, and reproduction in any medium, provided the original work is properly cited. 
morphism influences PON1 protein stability and concentration, while the $-108 \mathrm{C}>\mathrm{T}$ promoter polymorphism influences $P O N 1$ gene level of expression. ${ }^{9,13)}$

PON1 has an atheroprotective effect, mainly through the inhibition of low-density lipoproteins oxidation, which is the key process in the pathophysiology of atherosclerosis. ${ }^{14)}$ Indeed, low POase and AREase activities are associated with a greater risk for developing CVD. ${ }^{15)}$ Nonetheless, it remains unclear whether AAP could increase CVD risk by modulating PON1 activities. Studies reveal inconsistent data concerning the effects of AAP treatment on PON1 activities: while some studies describe a lowering or increasing effect of certain AAP on PON1 activities, others report no effect at all. ${ }^{16-18)}$ Curiously, none of these studies took into account the PON1 polymorphisms which could act as confounding factors, given the extreme interindividual variation of PON1 activities they can elicit. ${ }^{9}$

Although contradictory, evidence points to PON1 as a possible link between schizophrenia, AAP treatment and increased CVD risk. The objective of this study was to investigate the AREase and POase enzymatic activities of PON1, along with the assessment of PON1 gene polymorphisms in schizophrenic patients treated with either olanzapine or another AAP, as compared to healthy controls.

\section{METHODS}

\section{Participants}

We performed a case-control study on 60 schizophrenic patients and 34 healthy controls. Individuals with a history of any genetic, metabolic or neurologic condition, as well as those having a history of acute or chronic inflammatory disease or taking long-term medication (other than AAP in case of schizophrenic patients) were excluded from the study. The patients were diagnosed according to the Diagnostic and Statistical Manual of Mental Disorders, fifth edition (DSM-5) criteria for schizophrenia. ${ }^{19)}$ The schizophrenic patients were treated with either olanzapine $(n=27)$ or other AAP $(n=33)$. The healthy controls were selected during routine health checks and had no past or present history of psychiatric disorders. All participants were interviewed about their age, personal medical history and previous treatments. Informed consent from each participant and ethical com- mittee approval from luliu Hațieganu University of Medicine and Pharmacy (Cluj-Napoca, Romania) were obtained, the research protocol being in agreement with the Declaration of Helsinki of the World Medical Association.

\section{Blood Samples Collection and Preparation}

Venous blood samples were obtained from each participant and collected into vacutainers containing lithium heparin or ethylenediamine tetraacetic acid di-potassium salt, in order to obtain plasma and whole blood, respectively. The vacutainers containing lithium heparin were centrifuged for 5 minutes at 3,500 rpm and the plasma was collected, aliquoted and stored at $-80^{\circ} \mathrm{C}$ until analysis. The whole blood was processed for isolation and purification of genomic DNA, which was stored at $-20^{\circ} \mathrm{C}$ until analysis.

\section{Paraoxonase 1 Genotyping}

Genomic DNA was isolated from whole blood using the Wizard ${ }^{\mathbb{R}}$ Genomic DNA Purification kit and protocol (Promega, Madison, WI, USA). Genotyping of DNA samples was performed on Eppendorf Mastercycler thermal cycler (Eppendorf, Hamburg, Germany), using the polymerase chain reaction-restriction length polymorphism (PCR-RFLP) technique, adapted from Brophy et al. ${ }^{20)}$

\section{Paraoxonase 1 Activities}

PON1 AREase and $\mathrm{NaCl}$-stimulated paraoxonase (ssPOase) activities were measured spectrophotometrically at $25^{\circ} \mathrm{C}$, using a double beam ultraviolet-visible spectrophotometer (JASCO V-530; JASCO, Easton, MD, USA) according to the method described by Eckerson et $a .^{211}$

\section{Statistical Analysis}

Data were analyzed using the IBM SPSS Statistics (version 23; IBM Co., Armonk, NY, USA). All quantitative variables were tested for normal distribution using the Shapiro-Wilk test. Normally distributed data were presented as mean \pm standard deviation, while asymmetric data were described by median and range. Qualitative variables were presented using frequencies. Quantitative data between groups were compared by using the independent-samples $t$ test (for normally distributed data) and the Mann-Whitney $U$ test or Kruskal-Wallis test (for 
asymmetric data). In order to compare the frequencies of qualitative variables, Pearson's chi-square test was used.

The multiple regression analysis for the AREase and ssPOase activities was conducted by using six independent variables (gender, age, group, Q192R, M55L, and $-108 \mathrm{C}>\mathrm{T}$ polymorphisms). The linear regression analysis was performed in order to assess the influence of the three PON1 gene polymorphisms on AREase and ssPOase activities. No multiple comparisons adjustment was used in our study since we made a small number of planned comparisons with clearly defined hypotheses, avoiding post-hoc comparisons.

Table 1. Sociodemographic and clinical characteristics of the study participants

\begin{tabular}{lccc}
\hline \multicolumn{1}{c}{ Characteristic } & $\begin{array}{c}\text { Schizophrenic } \\
\text { patients }(\mathrm{n}=60)\end{array}$ & $\begin{array}{c}\text { Healthy } \\
\text { controls }(\mathrm{n}=34)\end{array}$ & $p$ value \\
\hline Gender & & & $0.001^{*}$ \\
Male & 42 & 12 & \\
Female & 18 & 22 & \\
Male/female ratio & 2.33 & 0.54 & \\
Age (yr) & $34.04 \pm 9.55$ & $39.79 \pm 11.42$ & $0.011^{*}$ \\
AAP & & - & - \\
Olanzapine & $27(45.0)$ & - & - \\
Other & $33(55.0)$ & - & - \\
\hline
\end{tabular}

Values are presented as number only, mean \pm standard deviation, or number (\%).

AAP, atypical antipsychotics.

*Groups significantly different.

Table 2. PON 1 polymorphisms distribution and allele frequency in schizophrenic patients as compared to healthy controls

\begin{tabular}{ccccc}
\hline PON1 genotype & $\begin{array}{c}\text { Schizophrenic } \\
\text { patient }(\mathrm{n}=60)\end{array}$ & $\begin{array}{c}\text { Healthy } \\
\text { control }(\mathrm{n}=34)\end{array}$ & $\chi^{2}$ & $p$ value \\
\hline Q192R & & & 1.876 & 0.391 \\
QQ & $32(53.3)$ & $23(67.6)$ & & \\
QR & $22(36.7)$ & $9(26.5)$ & & \\
RR & $6(10.0)$ & $2(5.9)$ & & \\
Q/R & $0.72 / 0.28$ & $0.8 / 0.2$ & 1.966 & 0.16 \\
L55M & & & 8.847 & $0.012^{*}$ \\
LL & $37(61.7)$ & $12(35.3)$ & & \\
LM & $16(26.7)$ & $19(55.9)$ & & \\
MM & $7(11.7)$ & $3(8.8)$ & & \\
L/M & $0.75 / 0.25$ & $0.63 / 0.37$ & 2.902 & 0.088 \\
108C $>$ T & & & 1.594 & 0.451 \\
CC & $15(25.0)$ & $11(32.4)$ & & \\
CT & $27(45.0)$ & $15(44.1)$ & & \\
TT & $13(21.7)$ & $4(11.8)$ & & \\
Undetermined & $5(8.3)$ & $4(11.8)$ & & \\
C/T & $0.52 / 0.48$ & $0.62 / 0.38$ & 1.523 & 0.217 \\
\hline
\end{tabular}

Values are presented as number (\%).

*Groups significantly different.
A confidence interval of $95 \%$ and two-tailed $p$ values of less than 0.05 were considered significant for all analyses.

\section{RESULTS}

\section{Characteristics of Participants}

Sociodemographic and clinical characteristics of the subjects included in the study are shown in Table 1. There were significant differences in gender distribution $(p=0.001)$ and age $(p=0.011)$ between groups.

\section{PON1 Polymorphisms Distribution and Allele Frequency}

PON1 polymorphisms distribution and allele frequency in schizophrenic patients as compared to healthy controls are shown in Table 2. All studied polymorphisms in patients and controls followed Hardy-Weinberg equilibrium ( $p>0.05)$. We found that Q192R and $-108 \mathrm{C}>\mathrm{T}$ polymorphisms distributions were similar between patients and controls. However, there was a significant difference between groups in L55M polymorphism distribution. Furthermore, there were no differences between groups in $\mathrm{Q} / \mathrm{R}, \mathrm{L} / \mathrm{M}$ and $\mathrm{C} / \mathrm{T}$ allele distribution.

\section{PON1 AREase Activity}

Schizophrenic patients $(n=60)$ had significantly lower $(-13.3 \%)$ AREase activity compared to the healthy controls $(n=34)(p=0.044)$, as shown in Table 3. Furthermore, taking into account the AAP medication, there was no significant difference in AREase activity between patients treated with olanzapine, patients treated with other AAP, and healthy controls, respectively $(p=0.131)$. However, schizophrenic patients treated with AAP had a lower

Table 3. PON1 AREase activity in schizophrenic patients compared to healthy controls

\begin{tabular}{ccccc}
\hline \multirow{2}{*}{ Subject } & \multicolumn{4}{c}{ AREase activity $(\mathrm{kU} / \mathrm{L})$} \\
\cline { 2 - 5 } & $\begin{array}{c}\text { Median } \\
\text { with IQR }\end{array}$ & Minimum & Maximum \\
\hline Schizophrenic patient $(\mathrm{n}=60)$ & 97.36 & $(34.7)^{*}$ & 42.4 & 215.3 \\
Olanzapine $(\mathrm{n}=27)$ & 99.57 & $(33.8)$ & 61.1 & 215.3 \\
Other AAP $(\mathrm{n}=33)$ & $95.55(35.1)$ & 42.4 & 160.2 \\
Healthy control $(\mathrm{n}=34)$ & $112.3(69.3)$ & 57.1 & 259.5 \\
\hline
\end{tabular}

PON1, paraoxonase 1; AREase, arylesterase; IQR, interquartile range; AAP, atypical antipsychotics.

${ }^{*} p=0.044$ (Mann-Whitney $U$ test), significantly different from healthy controls. 
Table 4. PON1 ssPOase activity in schizophrenic patients compared to healthy controls, according to the Q192R polymorphism distributions

\begin{tabular}{|c|c|c|c|c|}
\hline \multirow{3}{*}{ Subject } & \multicolumn{4}{|c|}{ ssPOase activity (U/L) } \\
\hline & \multicolumn{2}{|c|}{ QQ } & \multicolumn{2}{|c|}{$\mathrm{QR} / \mathrm{RR}$} \\
\hline & $\mathrm{n}$ & Median (range) & $\mathrm{n}$ & Median (range) \\
\hline Schizophrenic patient $(n=60)$ & 32 & $246.85(765.2)$ & 28 & $848.8(1,221.1)$ \\
\hline Olanzapine $(n=27)$ & 17 & $239.5(253.8)^{*}$ & 10 & $851.8(693.4)$ \\
\hline Other AAP $(n=33)$ & 15 & $271.4(704.9)$ & 18 & $847.25(1,221.1)$ \\
\hline Healthy control $(n=34)$ & 23 & $292.9(1,594.3)$ & 11 & $902.5(1,350.9)$ \\
\hline
\end{tabular}

PON1, paraoxonase 1; ssPOase, NaCl-stimulated paraoxonase; AAP, atypical antipsychotics.

${ }^{*} p=0.049$ (Mann-Whitney $U$ test), significantly different from other atypical antipsychotics-treated patients with QQ genotype and healthy controls with QQ genotype.

Table 5. Multiple regression analysis with AREase activity as dependent variable

\begin{tabular}{|c|c|c|c|c|c|}
\hline \multirow[t]{2}{*}{ Model } & \multicolumn{2}{|c|}{$\begin{array}{l}\text { Unstandardized } \\
\text { coefficients }\end{array}$} & \multirow{2}{*}{$\begin{array}{c}\begin{array}{c}\text { Standardized } \\
\text { coefficients }\end{array} \\
\beta\end{array}$} & \multirow[t]{2}{*}{$\mathrm{t}$} & \multirow{2}{*}{$p$} \\
\hline & B & SE & & & \\
\hline (Constant) & 108.68 & 22.82 & - & 4.762 & $<0.001$ \\
\hline Gender & 8.04 & 8.63 & 0.1 & 0.93 & 0.354 \\
\hline Age & -0.798 & 0.397 & -0.215 & -2 & 0.084 \\
\hline Group & -27.92 & 9.25 & -0.355 & -3.02 & $0.003 *$ \\
\hline Q192R & -0.27 & 7.06 & -0.004 & -0.038 & 0.97 \\
\hline L55M & 11.49 & 7.09 & 0.21 & 1.62 & 0.11 \\
\hline$-108 \mathrm{C}>\mathrm{T}$ & 8.94 & 6.5 & 0.16 & 1.37 & 0.173 \\
\hline
\end{tabular}

AREase, arylesterase; SE, standard error.

*Statistically significant, $p<0.05$.

AREase activity compared to healthy controls, either it was the subgroup treated with olanzapine $(-11.3 \%)$ or with other AAP $(-14.9 \%)$.

\section{PON1 ssPOase Activity}

Given the known influence of the Q192R polymorphism on the POase activity of PON1, we distributed the patients and control subjects into either $Q Q$ or $Q R / R R$ subgroups according to the Q192R polymorphism distributions, as shown in Table 4.

As expected, subjects with QQ genotype had significantly lower ( $-69.2 \%)$ ssPOase activity, compared to subjects with QR/RR genotypes ( $p<0.001)$.

Referring to the QQ genotype subgroup, there was no significant difference between schizophrenic patients ( $n=32$, regardless of their medication) and healthy controls $(\mathrm{n}=23)(p=0.067)$, as shown in Table 4. However, schizophrenic patients had a lower $(-15.7 \%)$ ssPOase activity compared to healthy controls. Taking into account the AAP medication, we found a significant differ-
Table 6. Multiple regression analysis with ssPOase activity as dependent variable

\begin{tabular}{lccccccc}
\hline \multirow{2}{*}{ Model } & \multicolumn{2}{c}{$\begin{array}{c}\text { Unstandardized } \\
\text { coefficients }\end{array}$} & \multicolumn{2}{c}{$\begin{array}{c}\text { Standardized } \\
\text { coefficients }\end{array}$} & $\mathrm{t}$ & $p$ \\
\cline { 2 - 3 } \cline { 7 - 8 } & \multicolumn{2}{c}{$\mathrm{B}$} & $\mathrm{SE}$ & & $\beta$ & & \\
\hline (Constant) & -439.62 & 145.51 & & - & -3.02 & 0.003 \\
Gender & 10.84 & 55.03 & & 0.14 & 0.197 & 0.844 \\
Age & -0.647 & 2.53 & & -0.017 & -0.255 & 0.799 \\
Group & -82.33 & 58.97 & & -0.1 & -1.4 & 0.167 \\
Q192R & 486.75 & 45.02 & & 0.769 & 10.81 & $<0.001^{*}$ \\
L55M & 28.61 & 45.2 & & 0.05 & 0.633 & 0.529 \\
-108C $>$ T & 128.83 & 41.47 & & 0.233 & 3.11 & $0.003^{*}$ \\
\hline
\end{tabular}

ssPOase, $\mathrm{NaCl}$-stimulated paraoxonase; $\mathrm{SE}$, standard error. *Statistically significant, $p<0.05$.

ence in ssPOase activity between olanzapine-treated patients, other AAP-treated patients and healthy controls, respectively $(p=0.049)$. Thus, olanzapine-treated patients had lower ssPOase activity compared to other AAP-treated patients $(-7.2 \%)$ and healthy controls $(-18.2 \%)$, respectively.

Regarding the QR/RR genotypes subgroup, there was no significant difference between schizophrenic patients ( $n=28$, regardless of their medication) and healthy controls $(\mathrm{n}=11)(p=0.59)$. However, schizophrenic patients had a lower $(-5.95 \%)$ ssPOase activity compared to healthy controls. Taking into account the AAP medication, there was no significant difference in ssPOase activity between olanzapine-treated patients, other AAPtreated patients, and healthy controls, respectively ( $p=$ 0.765). However, schizophrenic patients had a lower ssPOase activity compared to healthy controls, both for olanzapine $(-5.6 \%)$ and other AAP $(-6.1 \%)$ treated subgroups. 


\section{Regression Analysis}

We performed a multiple regression analysis, which showed that AREase activity was significantly influenced (adjusted $R^{2}=0.151, F_{6,74}=3.368, p=0.005$ ) only by group $(\beta=-0.355$, standard error $[\mathrm{SE}]=9.25, \quad p=0.003)$, as shown in Table 5.

Moreover, ssPOase activity was significantly influenced (adjusted $R^{2}=0.664, F_{6,74}=27.368, p<0.001$ ) by Q192R $(\beta=0.769, \mathrm{SE}=45.02, p<0.001)$ and $-108 \mathrm{C}>\mathrm{T}$ ( $\beta=0.233$, $\mathrm{SE}=41.47, \rho=0.003$ ) polymorphisms, as shown in Table 6.

We also performed a linear regression analysis, which showed that the Q192R polymorphism explained $64.6 \%$ of ssPOase activity total variance (adjusted $\mathrm{R}^{2}=0.646, p<$ 0.001), but did not influence AREase activity (adjusted $\left.\mathrm{R}^{2}=-0.011, p=0.868\right)$. Moreover, the L55M polymorphism explained $19.6 \%$ of ssPOase activity total variance (adjusted $\mathrm{R}^{2}=0.196, p<0.001$ ), but influenced to a lesser extent AREase activity (adjusted $R^{2}=0.038$, $p=0.033)$. Lastly, the $-108 \mathrm{C}>\mathrm{T}$ polymorphism had a modest influence on both ssPOase (adjusted $R^{2}=0.059$, $p=0.014$ ) and AREase (adjusted $R^{2}=0.072, p=0.008$ ) activities.

\section{DISCUSSION}

Recent evidence suggests that PON1 plays a role in the association between schizophrenia, AAP treatment and increased CVD risk. In the current study, we aimed at investigating the PON1 status in schizophrenic patients treated with either olanzapine or other AAP. Thus, we assessed the most common polymorphisms in the PON1 gene (Q192R, L55M, and $-108 \mathrm{C}>\mathrm{T}$ ) along with PON1 activities (AREase and ssPOase).

PON1 polymorphisms account for the great interindividual variation of $\mathrm{PON} 1$ concentration and activities. ${ }^{9)}$ Our data showed that PON1 polymorphisms distribution and allele frequency in our participants were similar to those in other European populations. ${ }^{22)}$ Q192R and $-108 \mathrm{C}>\mathrm{T}$ polymorphisms distribution was similar between patients and controls. However, there was a significant difference in L55M polymorphism distribution between groups, explained by a higher LL genotype frequency in patients as compared to controls. The L55M polymorphism influences PON1 protein stability and concentration and has a significant effect on PON1 activities independent of Q192R polymorphism. ${ }^{9,13,23)}$ Indeed, our findings showed that L55M polymorphism significantly influenced both AREase and ssPOase activities. Although some studies suggest that LL genotype might be a protective factor for schizophrenia, our data did not endorse this hypothesis. ${ }^{24)}$

Recent investigations indicate that low AREase and POase activities are associated with greater risk for developing CVD. ${ }^{15)}$ However, it remains unclear whether AAP could increase CVD risk by altering PON1 activities. Our data showed that AAP-treated patients had significantly reduced AREase activity. As low AREase activity predicts $\mathrm{CVD}$, it may be assumed that AAP-treated patients are exposed to a greater risk for CVD. ${ }^{15)}$ Since AREase activity is considered a reliable indicator of PON1 concentration, this could also indicate a reduced level of the protein in AAP-treated patients. ${ }^{12)}$ However, patients had significantly higher LL genotype frequency, which is associated with elevated levels of PON1. ${ }^{13)}$ Thus, there might be another factor that can account for the observed low AREase activity.

Furthermore, we assessed the ssPOase activity in relation to Q192R polymorphism. The Q192R polymorphism has a marked influence on the POase activity, eliciting a low activity in subjects with QQ genotype and high activity in subjects with QR and RR genotypes. ${ }^{9,11)}$ Indeed, our findings were in line with these observations. Furthermore, concerning the subjects with QQ genotype, we found significantly reduced ssPOase activity in the subgroup of schizophrenic patients treated with olanzapine. The subgroup of subjects with QR and RR genotypes had similar ssPOase activities. As low POase activity predicts $\mathrm{CVD}$, it may be assumed that olanzapine-treated patients with QQ genotype are exposed to the greatest risk for CVD. ${ }^{15)}$ Indeed, among AAP, olanzapine has the highest potential of adverse metabolic effects and subsequently CVD. ${ }^{4,5)}$ Nevertheless, there is no clear association between individuals with QQ genotype and increased CVD risk. ${ }^{15)}$ Therefore, it may be assumed that subjects with QQ genotype, having a genetically determined lower POase activity are more vulnerable to the effect of olanzapine. Conversely, the observed low POase activity could be the consequence of a low PON1 protein level, as the AREase activity was also lower in schizophrenic patients. However, the LL genotype, associated with ele- 
vated levels of PON1, was also more frequent in patients. ${ }^{13)}$ Thus, there might be a common factor that can decrease both AREase and POase activities.

As studies reveal inconsistent data, the influence of AAP treatment on PON1 activities remains to be determined. Nonetheless, the observed low PON1 activities might result from the combined effect of PON1 polymorphic variants, other interfering genes, and environmental factors. Intra- and inter-assay variation for PON1 activities were $<5 \%$, so the observed differences in activities cannot be explained by technical variability. The multiple regression analysis performed in our participants showed that AREase activity was influenced only by group, whereas the major determinants of ssPOase activity were Q192R and - 108C > T polymorphisms, as previously reported in other studies., ${ }^{9,15)}$

Studies report conflicting data regarding the effects of AAP treatment on PON1 activities. One study did not find any significant effect of olanzapine on PON1 activities. ${ }^{18)}$ However, other study reported significantly lower POase activity in patients treated with olanzapine, compared with patients treated with quetiapine and healthy controls. ${ }^{16)}$ Conversely, another study reported an increasing effect of the AAP drug risperidone on AREase activity. ${ }^{17)}$ All these observations should be regarded with caution, as each AAP might have varying effects on PON1 activities. Moreover, none of these studies took into account the significant influence of PON1 polymorphisms on activities. To the best of our knowledge, this is the first study that reports on PON1 activities in AAP-treated schizophrenic patients, in relation to PON1 polymorphisms distribution.

Whereas our study has reached its aims, it had several limitations. First, our groups were unequal in size, due to difficulties in recruiting healthy peers. Second, our studied groups were not gender- and age-matched. Although there was a significant difference in gender and age between groups, neither of these factors significantly influenced PON1 activities, as shown by our multiple regression analysis. Finally, we lacked information on several factors, such as duration of the psychiatric illness, body mass index (BMI), lipid profile, or lifestyle (smoking, eating habits and exercise), all of which could have influenced PON1 activities. ${ }^{25)}$

In order to clarify the PON1 role in the association between schizophrenia, AAP treatment and increased CVD risk, further research is needed. Studies should emphasize the effects of each AAP on PON1 activities, since each AAP has a unique risk profile. ${ }^{26)}$ It is possible to compare different antipsychotic treatments, given that the AAP dosage and duration of treatment can be transformed to chlorpromazine or haloperidol equivalents. ${ }^{27)}$ Ideally, one should assess PON1 activities before and after AAP treatment, in order to discard any effects elicited by the psychiatric condition. At the same time, one should control for other factors influencing PON1 activities, such as PON1 gene polymorphisms, lipid profile, BMI and lifestyle.

In summary, this is the first study that reports on low PON1 activities in AAP-treated schizophrenic patients, in relation to PON1 polymorphisms distribution. These results provide some possible explanation for the increased risk of CVD in schizophrenic patients treated with AAP. Also, whether the QQ genotype is a possible biomarker for such increased risk in schizophrenic patients receiving AAP will require more extensive investigations.

\section{REFERENCES}

1. Bruijnzeel D, Suryadevara U, Tandon R. Antipsychotic treatment of schizophrenia: an update. Asian J Psychiatr 2014; 11:3-7.

2. Haro JM, Salvador-Carulla L. The SOHO (Schizophrenia Outpatient Health Outcome) study: implications for the treatment of schizophrenia. CNS Drugs 2006;20:293-301.

3. Yogaratnam J, Biswas N, Vadivel R, Jacob R. Metabolic complications of schizophrenia and antipsychotic medications-an updated review. East Asian Arch Psychiatry 2013; 23:21-28.

4. Sarandol A, Kirli S, Akkaya C, Ocak N, Eroz E, Sarandol E. Coronary artery disease risk factors in patients with schizophrenia: effects of short term antipsychotic treatment. I Psychopharmacol 2007;21:857-863.

5. Janicak PG. Metabolic syndrome. In: Schizophrenia: recent advances in diagnosis and treatment. New York, NY: Springer;2014. p.239-240.

6. Hennekens $\mathrm{CH}$, Hennekens AR, Hollar D, Casey DE. Schizophrenia and increased risks of cardiovascular disease. Am Heart J 2005; 150:1115-1121.

7. Costa LG, Vitalone A, Cole TB, Furlong CE. Modulation of paraoxonase (PON1) activity. Biochem Pharmacol 2005; 69:541-550

8. Gan KN, Smolen A, Eckerson HW, La Du BN. Purification of human serum paraoxonase/arylesterase. Evidence for one esterase catalyzing both activities. Drug Metab Dispos 1991;19:100-106. 
9. Chen J, Chan W, Wallenstein S, Berkowitz G, Wetmur JG. Haplotype-phenotype relationships of paraoxonase-1. Cancer Epidemiol Biomarkers Prev 2005; 14:731-734.

10. Mackness M, Mackness B. Human paraoxonase-1 (PON1): Gene structure and expression, promiscuous activities and multiple physiological roles. Gene 2015;567:12-21.

11. Mackness B, Mackness MI, Arrol S, Turkie W, Julier K, Abuasha B, et al. Serum paraoxonase (PON1) 55 and 192 polymorphism and paraoxonase activity and concentration in non-insulin dependent diabetes mellitus. Atherosclerosis 1998;139:341-349.

12. Richter RJ, Jarvik GP, Furlong CE. Determination of paraoxonase 1 status without the use of toxic organophosphate substrates. Circ Cardiovasc Genet 2008;1:147-152.

13. Garin MC, James RW, Dussoix $\mathrm{P}$, Blanché $\mathrm{H}$, Passa $\mathrm{P}$, Froguel $\mathrm{P}$, et al. Paraoxonase polymorphism Met-Leu54 is associated with modified serum concentrations of the enzyme. A possible link between the paraoxonase gene and increased risk of cardiovascular disease in diabetes. J Clin Invest 1997;99:6266.

14. Précourt LP, Amre D, Denis MC, Lavoie JC, Delvin E, Seidman $\mathrm{E}$, et al. The three-gene paraoxonase family: physiologic roles, actions and regulation. Atherosclerosis 2011,214:20-36.

15. Gupta N, Singh S, Maturu VN, Sharma YP, Gill KD. Paraoxonase 1 (PON1) polymorphisms, haplotypes and activity in predicting CAD risk in North-West Indian Punjabis. PLoS One 2011;6:e17805.

16. Unsal C, Albayrak Y, Albayrak N, Kuloglu M, Hashimoto K. Reduced serum paraoxonase 1 (PON1) activity in patients with schizophrenia treated with olanzapine but not quetiapine. Neuropsychiatr Dis Treat 2013;9:1545-1552.

17. Noto C, Ota VK, Gadelha A, Noto MN, Barbosa DS, Bonifácio $\mathrm{KL}$, et al. Oxidative stress in drug naïve first episode psychosis and antioxidant effects of risperidone. I Psychiatr Res 2015;68:210-216.

18. Camkurt MA, Gunes M, Demir S, İbiloglu A, Kaya MC, Bulut
M, et al. Serum paraoxonase levels of schizophrenia patients treated with typical, atypical and combined antipsychotics. Bull Clin Psychopharmacol 2015;25(Suppl 1):S110.

19. American Psychiatric Association. Schizophrenia 295.90 (F20.9). In: Diagnostic and statistical manual of mental disorders: DSM-5. 5th ed. Arlington, VA:American Psychiatric Publishing;2013.

20. Brophy VH, Jampsa RL, Clendenning JB, McKinstry LA, Jarvik $\mathrm{GP}$, Furlong CE. Effects of $5^{\prime}$ regulatory-region polymorphisms on paraoxonase-gene (PON1) expression. Am J Hum Genet 2001;68:1428-1436.

21. Eckerson HW, Wyte CM, La Du BN. The human serum paraoxonase/arylesterase polymorphism. Am J Hum Genet 1983; 35:1126-1138.

22. La Du BN. Human serum paraoxonase/arylesterase. In: Kalow $W$, editor. Pharmacogenetics of drug metabolism. New York, NY:Pergamon Press; 1992. p.51-91.

23. Mackness B, Mackness MI, Arrol S, Turkie W, Durrington PN. Effect of the molecular polymorphisms of human paraoxonase (PON1) on the rate of hydrolysis of paraoxon. Br J Pharmacol 1997; 122:265-268.

24. Kucukali Cl, Aydin M, Ozkok E, Orhan N, Cakir U, Kilic G, et al. Paraoxonase-1 55/192 genotypes in schizophrenic patients and their relatives in Turkish population. Psychiatr Genet 2008; 18:289-294.

25. Deakin SP, James RW. Genetic and environmental factors modulating serum concentrations and activities of the antioxidant enzyme paraoxonase-1. Clin Sci (Lond) 2004;107:435447 .

26. Melkersson K, Dahl ML. Adverse metabolic effects associated with atypical antipsychotics: literature review and clinical implications. Drugs 2004;64:701-723.

27. Andreasen NC, Pressler M, Nopoulos P, Miller D, Ho BC. Antipsychotic dose equivalents and dose-years: a standardized method for comparing exposure to different drugs. Biol Psychiatry 2010;67:255-262. 